\title{
Influence of solitons on the transition to spatiotemporal chaos in coupled map lattices
}

Mikkelsen, R.; van Hecke, M.; Bohr, Tomas

Published in:

Physical Review E. Statistical, Nonlinear, and Soft Matter Physics

Link to article, DOI:

10.1103/PhysRevE.67.046207

Publication date:

2003

Document Version

Publisher's PDF, also known as Version of record

Link back to DTU Orbit

Citation (APA):

Mikkelsen, R., van Hecke, M., \& Bohr, T. (2003). Influence of solitons on the transition to spatiotemporal chaos in coupled map lattices. Physical Review E. Statistical, Nonlinear, and Soft Matter Physics, 67(4), 046207. https://doi.org/10.1103/PhysRevE.67.046207

\section{General rights}

Copyright and moral rights for the publications made accessible in the public portal are retained by the authors and/or other copyright owners and it is a condition of accessing publications that users recognise and abide by the legal requirements associated with these rights.

- Users may download and print one copy of any publication from the public portal for the purpose of private study or research.

- You may not further distribute the material or use it for any profit-making activity or commercial gain

- You may freely distribute the URL identifying the publication in the public portal 


\title{
Influence of solitons on the transition to spatiotemporal chaos in coupled map lattices
}

\author{
René Mikkelsen, ${ }^{1,2}$ Martin van Hecke, ${ }^{3}$ and Tomas Bohr ${ }^{4}$ \\ ${ }^{1}$ Center for Chaos and Turbulence Studies, The Niels Bohr Institute, Blegdamsvej 17, DK-2100 Copenhagen D, Denmark \\ ${ }^{2}$ Department of Applied Physics and J.M. Burgers Centre for Fluid Dynamics, University of Twente, P.O. Box 217, 7500 AE Enschede, \\ The Netherlands \\ ${ }^{3}$ Kamerlingh Onnes Laboratory, University of Leiden, Niels Bohrweg 2, 2333 CA Leiden, The Netherlands \\ ${ }^{4}$ Department of Physics, The Danish Technical University, DK-2800 Kgs. Lyngby, Denmark \\ (Received 8 July 2002; revised manuscript received 18 November 2002; published 14 April 2003)
}

\begin{abstract}
We study the transition from laminar to chaotic behavior in deterministic chaotic coupled map lattices and in an extension of the stochastic Domany-Kinzel cellular automaton [E. Domany and W. Kinzel, Phys. Rev. Lett. 53, 311 (1984)]. For the deterministic coupled map lattices, we find evidence that "solitons" can change the nature of the transition: for short soliton lifetimes it is of second order, while for longer but finite lifetimes, it is more reminiscent of a first-order transition. In the second-order regime, the deterministic model behaves like directed percolation with infinitely many absorbing states; we present evidence obtained from the study of bulk properties and the spreading of chaotic seeds in a laminar background. To study the influence of the solitons more specifically, we introduce a soliton including variant of the stochastic Domany-Kinzel cellular automaton. Similar to the deterministic model, we find a transition from second- to first-order behavior due to the solitons, both in a mean-field analysis and in a numerical study of the statistical properties of this stochastic model. Our study illustrates that under the appropriate mapping some deterministic chaotic systems behave like stochastic models; but it is hard to know precisely which degrees of freedom need to be included in such description.
\end{abstract}

DOI: 10.1103/PhysRevE.67.046207

\section{INTRODUCTION}

Spatiotemporal chaos occurs in many spatially extended deterministic systems and remains notoriously difficult to characterize [2]. Therefore, one may attempt to map such deterministic chaotic systems onto stochastic models for which many more analytical methods are available. It is then tacitly assumed that, after sufficient coarse graining of the deterministic model, the role of deterministic chaos can be taken over by the noise in the stochastic system. A critical test of the validity of such mappings are the predictions for the transitions between qualitatively different states that extended chaotic systems display. The key question is then as follows: Are transitions in deterministic chaotic systems governed by the universality classes of stochastic systems?

As is known for a variety of spatiotemporal chaotic systems $[2,3]$ and as we will show below for the deterministic system at hand, chaotic states in extended systems often display a mixture of rather regularly propagating structures and more disordered behavior. When the propagating structures, that we will refer to as "solitons" (following Ref. [4]) have a finite lifetime, it may seem that they can be ignored after sufficient coarse graining. We will find strong indications that this is not always the case, and we will give an example where their influence may even be so strong as to change the nature of the transition. We will also show that extending simple stochastic models with the appropriate solitonic degrees of freedom can mimic this behavior quite accurately: not only can we change the order of the transition, we can also get transient nonuniversal scaling of the type observed in coupled map lattices [5]. Therefore, we conclude that, in many cases, deterministic chaotic systems can indeed be mapped to stochastic models. A short account of our work
PACS number(s): 05.45.Jn, 05.70.Jk, 47.27.Cn, 05.45.Ra

has already been published [6].

\section{A. Historical background}

Chaté and Manneville $[7,8]$ introduced the notion of a universal transition to extended chaos via "spatiotemporal intermittency" (STI) in a study of the deterministic damped Kuramoto-Sivashinsky partial differential equation [9]. STI states are composed of "turbulent" (chaotic) and "laminar" (ordered) patches, and the laminar patches remain so except for contamination by turbulence at their boundaries. These states are conjectured to occur quite generally when, locally, laminar and turbulent dynamics are separated by a subcritical bifurcation, and indeed a large number of different experimental systems and theoretical models display STI [10].

As a function of their parameters, STI systems display a transition from states where the turbulence eventually dies out to states where the turbulence spreads and dominates. Pomeau proposed [11] an analogy between this transition and the phase transition of the stochastic process known as directed percolation (DP); for an introduction to DP, see, e.g., Refs. [12,13]. In directed percolation, one considers the spreading of "activity" in an absorbing, inactive background. Earlier, Grassberger [14] and Janssen [15] had conjectured that any stochastic process with an unique absorbing state should be in the same directed percolation universality class.

Relating laminar to inactive and turbulent to active states appears to map spatiotemporal intermittency to directed percolation. To verify whether deterministic chaotic models with an absorbing state would be in the DP universality class, Chaté and Manneville introduced a very simple coupled map lattice (CML) that displays STI and numerically obtained the 
critical exponents that characterize the transition from inactive to active states. Surprisingly, these critical exponents appear to vary with the parameters and are in general different from the DP values. Therefore, the Chaté-Manneville model appears to be not in the DP universality class and not even universal.

Grassberger and Schreiber [4] pointed out that the presence of long lived traveling structures, which they call solitons in the Chaté-Manneville model, may lead to large crossover times, and conjectured that in the long-time limit the behavior of the Chate-Manneville model would be in the DP universality class.

Recently, the Chate-Manneville model with an asynchronous update rule was studied [16]. Here random sites are chosen to be iterated forward while keeping the others unaltered. For this model, the solitons observed for the standard synchronous update rule are suppressed and the critical exponents are universal with DP values, implying that the Chaté-Manneville model with asynchronous updating belongs to the DP universality class. However, the asynchronous updating introduces an element of stochasticity into the model, thus ruining the deterministic character of the original model.

\section{B. Outline}

In this paper, we will study a deterministic extension of the Chate-Manneville CML that facilitates the tuning of the soliton properties. We will demonstrate that the influence of solitons may be much more profound than setting a crossover time, since they appear to be able to change the type of transition from second to first order. The role of the solitons is further illustrated in an extension of the stochastic Domany-Kinzel cellular automaton. In its standard form, all sites of this model can be either active or inactive, but we will add a "solitonic" degree of freedom that mimics the behavior of the solitons in the CMLs. The mean-field equations of this stochastic model show a transition from secondorder DP-like behavior to a first-order transition when the soliton lifetimes are increased. Numerical studies of this stochastic model also find evidence for such a crossover to firstorder behavior, although it is very difficult to asses the asymptotic behavior for our model. In any case, we present strong numerical evidence that the transition is not an ordinary second-order transition and that there is no asymptotic scaling regime, although there are appears to be a transient that displays nonuniversal scaling behavior.

Our study illustrates that for extended systems, it is a difficult task to faithfully map a deterministic system to a stochastic counterpart. In this particular case, localized propagating structures can be identified as responsible for the breakdown of DP universality, but one can imagine that less easily identified properties of the deterministic dynamics could be responsible for such a breakdown in other systems.

The outline of this paper is as follows. In Sec. II, we discuss the coupled map lattices. Starting from a brief discussion of the classic Chate-Manneville model, we introduce our extension to lattices of two-dimensional maps in Sec. II A. We show that the new parameter, that is introduced, has a profound effect on the importance of "solitons," and that long living solitons change the transition from inactive to active states from a second- to a first-order transition in Sec. II B. In the second-order regime, we estimate the bulk critical exponents using finite size scaling techniques in Sec. II C, and measure spreading exponents in Sec. II D. All this data is consistent with the coupled map lattice being in the universality class of directed percolation with infinitely many absorbing states, provided that soliton lifetimes are short. In Sec. III, we discuss the extension of the standard DomanyKinzel cellular automata which includes new degrees of freedom that mimic the solitons of the coupled map lattices. The mean-field equations for this model are studied in Sec. III B, and these show a transition from second- to first-order behavior as a function of the soliton lifetimes. We study the phenomenology and its statistical bulk properties of the full model in the soliton rich regime in Sec. III C. The behavior of the model in the soliton rich regime is quite distinct from an ordinary second-order transition.

\section{COUPLED MAP LATTICES}

The model introduced by Chaté and Manneville consists of coupled maps, each of which either performs "laminar" or chaotic motion. The model was motivated by the fact that studies of the deterministic partial differential equations, such as the damped Kuramoto-Sivashinsky equation, are numerically quite demanding and had not provided enough precision to allow a definitive comparison to DP $[5,7]$. In one spatial dimension, their coupled map lattice was defined according to

$$
u_{i}(n+1)=f\left(u_{i}(n)\right)+\frac{\varepsilon}{2} \Delta_{f} u_{i}(n),
$$

where the subscripts $i$ denote the spatial position, $n$ is the discrete time and $\Delta_{f} u_{i}(n)=f\left(u_{i-1}(n)\right)-2 f\left(u_{i}(n)\right)$ $+f\left(u_{i+1}(n)\right)$. This expression is a discrete approximation of diffusive coupling in one dimension and introduces spatial correlations in the system; the parameter $\varepsilon$ is a measure of the coupling strength between a site $i$ and its two nearest neighbors at sites $(i-1)$ and $(i+1)$.

The map $f$ is chosen such that locally the scalar field $u_{i}$ can be in either of two states: the absorbing (laminar) or the chaotic (turbulent) one. When $u<1, f$ is a standard tent map of the form $f(u)=r\left(\frac{1}{2}-\left|u-\frac{1}{2}\right|\right)$ that displays chaotic behavior, while in the region where $u>1, f$ is simply the identity and leads to a laminar state. The sharp discontinuity in $f$ ensures that the two states are distinguishable at each site. The parameter $r>2$ determines the steepness of the tent map as well as the transition ratio from the chaotic to the laminar regime in the absence of coupling.

The form of the diffusive coupling ensures that turbulent sites cannot be spontaneously generated in a background of laminar sites: states where all sites are laminar remain so, and the laminar state is truly absorbing. The laminar state is not unique: Updating a state where all sites are in the laminar regime $\left(u_{i}>1\right)$ leads, via the diffusion operator, to a state where all variables are equal to the global average value $\bar{u}$. 
Once initiated, turbulent activity can spread through this CML by infecting laminar patches from their boundaries. The effectiveness of the resulting spreading of the chaos depends on the values of $r$ and $\varepsilon$. Suppose we study the behavior of this system by keeping $r$ fixed while varying the coupling strength $\varepsilon$. Completely analogous to DP, a critical value $\varepsilon=\varepsilon_{c}(r)$ exists, such that for $\varepsilon<\varepsilon_{c}$ an absorbing state is reached with unit probability, while sustained chaotic behavior (in the thermodynamic limit) is found for $\varepsilon>\varepsilon_{c}$. Taking the density of chaotic sites or "activity" $m$ as an order parameter, transitions from a "laminar" state (where $m$ decays to zero) to a "turbulent" state (where $m$ reaches a finite value in an infinite system) can be studied.

\section{A. Extensions to two-dimensional maps}

Coupled map lattices can, in principle, be related to continuous time physical systems of weakly coupled elements by interpreting the map $f$ as a return map on a Poincaré section. The time spent by two different sites between successive returns would in general be different for systems without periodic external forcing, and this was precisely the motivation for the asynchronous update rule in Ref. [16]. However, here we wish to mimic the variations in return times in a deterministic fashion. This motivated us to introduce a second field in the CML. Note that the simplest chaotic oscillator would be a system of three phase space dimensions like the Lorenz equations. Applying a Poincaré section reduces such a system to a two-dimensional map. This is also the case in systems with external periodic forcing. Here the simplest realization would be systems like a damped nonlinear pendulum or Duffing oscillator with a time-periodic forcing. A Poincaré section again reduces the system to a twodimensional map and after the synchronous iteration the respective units are still at equal time.

We, therefore, replace the single variable map $f(u)$, used in Eq. (1), by a new map with an additional variable $v$ :

$$
\begin{gathered}
u_{i}(n+1)=f\left(u_{i}(n)\right)+\frac{\varepsilon}{2} \Delta_{f} u_{i}(n)+v_{i}(n), \\
v_{i}(n+1)=b\left(u_{i}(n+1)-u_{i}(n)\right) .
\end{gathered}
$$

Here $f$ is the same map as before and the new parameter $b$ is the Jacobian of the full two-dimensional local map; this map is invertible for any nonzero $b$ and becomes increasingly two-dimensional with $|b|$. The change in the local map (1) is analogous to how the two-dimensional (2D) Hénon map [17] is constructed from the 1D logistic map, except that $b\left(u_{i}(n\right.$ $\left.+1)-u_{i}(n)\right)$ appears here on the right-hand side instead of $b u_{i}(n)$. This ensures that the absorbing state fixed points $u_{i}(n)=u^{*}$ of the old CML (1) are mapped to the laminar fixed point $\left(u_{i}(n), v_{i}(n)\right)=\left(u^{*}, 0\right)$ in the new CML. The model, Eqs. (2 and 3), is a completely deterministic system with no element of stochasticity and is updated synchronously. The value of $u_{i}$ determines, as in model (1), whether a given site is "active" or "inactive." Starting from the Chaté-Manneville case $(b=0)$, we can follow the transition between laminar and chaotic states. As we will see below, the
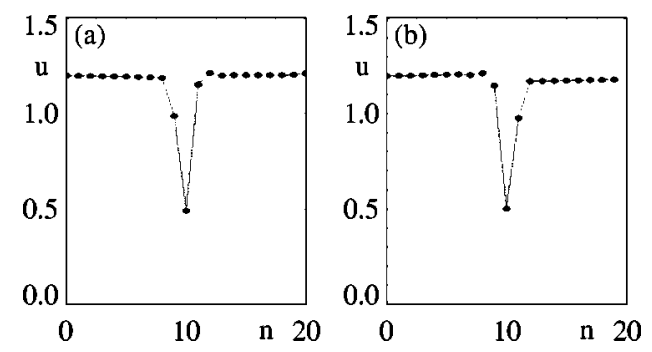

FIG. 1. Average profiles of a right (a) and left (b) moving soliton that occurs in the Chate-Manneville model at criticality for $r=3$.

new parameter $b$ actually opens up the possibility to study the effect of the solitons on the dynamical states and transitions of CMLs; this appears to be a more important issue than the dimensionality of the local map.

\section{B. Qualitative properties}

Our CML now contains three freely adjustable parameters $r, \varepsilon$, and $b$, and clearly we will have to focus on a subset of parameters. Our main focus will be on the case where $r$ $=3$, although we will also study the transition for $r=2.2$. For $r=3$ and $b=0$, the dynamics shows many solitons (see Fig. 1) and the critical exponents appear to differ significantly from those of DP.

To get a feeling for the location of the transition as function of $b$ and $\varepsilon$, we show in Fig. 2 the activity (defined as the average number of active sites) after 1000 iterations in the ranges $-0.3 \leqslant b \leqslant 0.3$ and $0 \leqslant \varepsilon \leqslant 0.4$ at $r=3.0$. The "traditional" transition is that occurring at $b=0$ and $\varepsilon$ $=0.35984 \ldots$ Clearly, for negative values of $b$, two additional transitions emerge. Here we only study points on the two transition branches labeled " $A$ " and " $B$ " in Fig. 2; below we focus on the behavior along branch A.

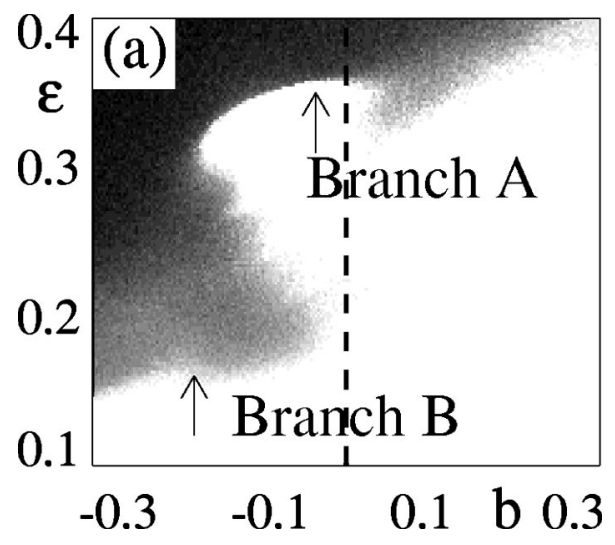

FIG. 2. Activity in the model, Eqs. (2) and (3), at $t=1000$. White regions correspond to points in the $(\varepsilon, b)$ plane where the initial activity has decayed into an absorbing configuration and the darker regions to points with a nonvanishing order parameter. Clearly the transition curve becomes quite complicated; the two branches discussed in this paper are indicated as "Branch $A$ " and "Branch B" (see text). The dashed line indicates the ChatéManneville model $(b=0)$. 


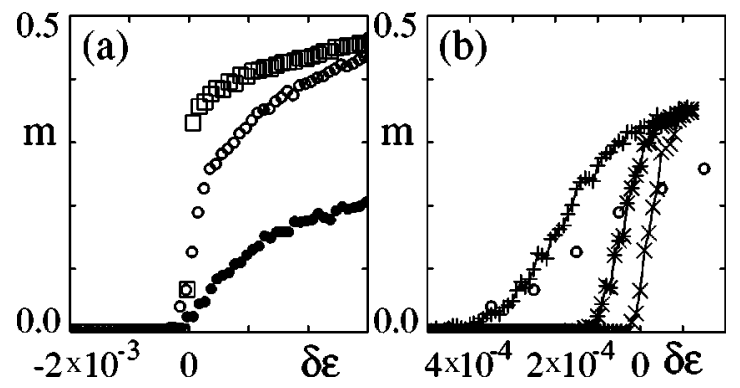

FIG. 3. Activity in the model, Eqs. (2) and (3), for 128 systems of size $L=2048$ and $r=3$. (a) Activity as a function of $\delta \varepsilon$ (distance to the critical point) at time $2 \times 10^{5}$ for $b=-0.1$ (squares), $b=0$ (open circles), and $b=0.2$ (closed circles). The transition appears much sharper for negative values of $b$. (b) Steepening for the transition at $b=-0.1$ for increasing times: $5 \times 10^{3}(+), 5 \times 10^{4}(*)$, and $5 \times 10^{5}(\times)$. To stress the magnified scale of $\delta \varepsilon$, also the data shown in panel (a) for $b=0$ is plotted (open circles).

\section{Qualitative changes in behavior along branch A}

Figure 2 hints that the sharpness of the transition varies along branch A: the jump in order parameter appears to become steeper for negative values of $b$. The differences in the nature of the transitions are illustrated more clearly in Fig. 3, by plotting the value of the order parameter as a function of $\varepsilon$ for $b=0$ and $b=-0.1$ averaged over an ensemble of 32 systems for a number of times. The behavior for $b=0$ is consistent with a continuous transition, whereas for $b$ $=-0.1$, longer times lead to a marked steepening, consistent with the emergence of a discontinuity.

Soliton regime. Some effects of the parameter $b$ on the dynamics can also be seen from the evolution of the binary patterns at $r=3$ (Fig. 4). For $b=0$, solitons can be seen both above and below threshold [Figs. 4(c)-4(d)]. They consist of pairs of active sites and propagate with velocity one. Their maximal lifetimes are of order 100 [Fig. 4(d)]. When $b$ is decreased to a value of -0.1 , the typical lifetimes of solitons become so long that they typically only vanish when they collide with other solitons or propagate into turbulent structures. When two solitons collide, they either annihilate or create new turbulent structures. Such creation is clearly visible in Fig. 4(a) for $n \approx 200$ and $i \approx 600$.

For sufficiently large $b$, the isolated solitons present in the original model $(b=0)$ are suppressed: solitons with a lifetime longer than a few iterations are rare here. On the other hand, there are regular "edge" states visible, where an active state propagates ballistically while emitting new activity; one example is visible in Fig. 4(e) for $n \approx 400$ and $i \approx 800$. These structures do not seem to influence the order of the transition, but they may very well lead to rather large crossover scales.

In conclusion, the value of $b$ has a large influence on the presence of solitons, and also influences the steepness of the transition. In fact, discontinuities are found at points in $(\varepsilon, b)$ space where solitons dominate the dynamics. This implies that the (colliding) solitons have a strong influence on the global dynamics and are able to change the nature of the transition from a continuous to what appears as a first-order one. We will make this point more precise below in our study of a stochastic model.
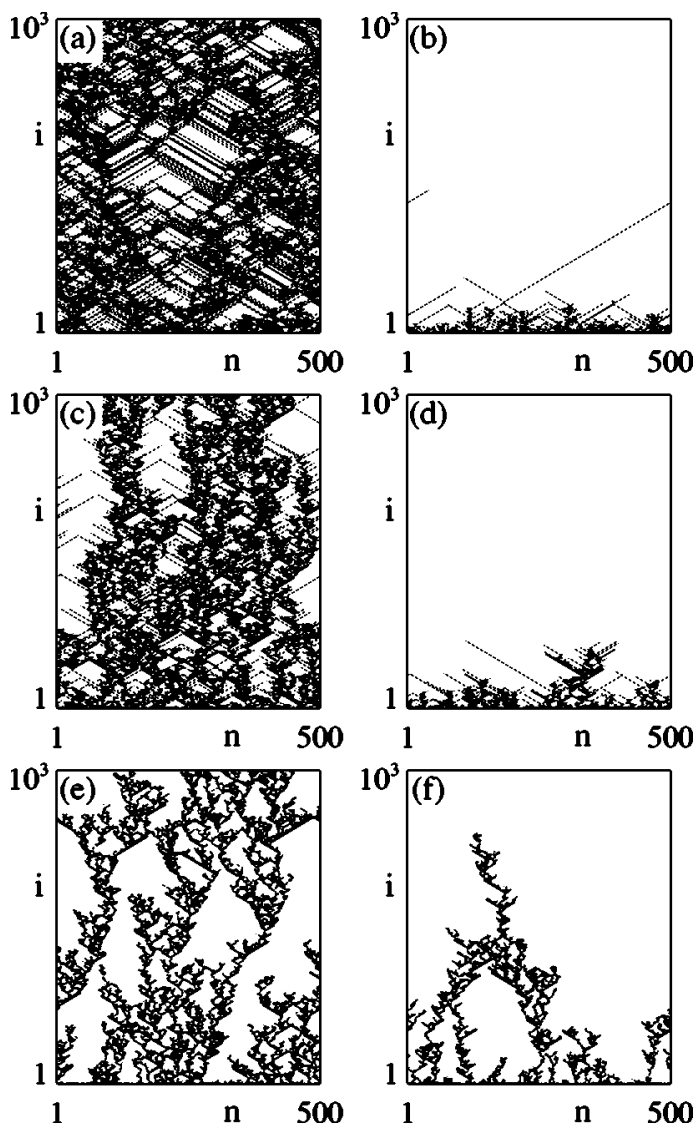

FIG. 4. Spacetime plots of our coupled map lattice Eqs. (2) and (3) for $r=3$ above (left column) and below (right column) criticality. Inactive sites are white, chaotic sites are black. (a) $b=-0.1$, $\varepsilon=0.353$; (b) $b=-0.1, \varepsilon=0.343$; (c) $b=0, \varepsilon=0.361$; (d) $b=0$, $\varepsilon=0.351$; (e) $b=0.2, \varepsilon=0.374$; and (f) $b=0.2, \varepsilon=0.364$.

\section{Finite size scaling in second-order regime}

Stochastic systems belonging to the DP universality class are characterized by a set of critical exponents describing, e.g., the order parameter $m(\varepsilon, L, t)$ and the behavior of the "absorption time" $\tau(r, \varepsilon, L)$, i.e., the averaged time it takes the system, starting from a random initial state, to reach the absorbing state. From finite-size scaling arguments [18], one finds that the order parameter $m$ at the critical point $\varepsilon_{c}$ should behave as

$$
m(L, t) \sim L^{-\beta / \nu_{\perp}} g\left(t / L^{z}\right) .
$$

For a finite lattice, the absorption time $\tau$ then increases as

$$
\tau \sim L^{z}
$$

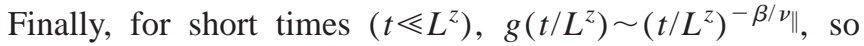
that for short times $m$ should decay as

$$
m(L, t) \sim t^{\theta} \text { for } t \ll L^{z}
$$

Here the usual dynamical exponent $z=\nu_{\|} / \nu_{\perp}$ has been introduced, defined as the ratio between the correlation length 

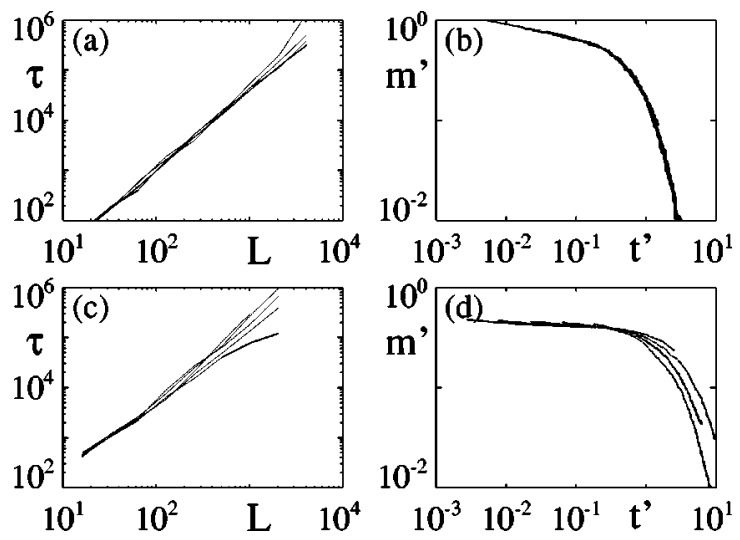

FIG. 5. Examples of good rescaling plots for $b=0.2$ [(a) and (b)] and poor rescaling for $b=-0.1[$ (c) and (d)]. (a) Absorption time $\tau$ vs system size $\mathrm{L}$, for $r=3, b=0.2$ and $\varepsilon=0.3727,0.37322$, 0.37323 (critical value), $0.37325,0.3733$, and 0.3735. (b) Rescaled average activity $m^{\prime}:=m L^{-\theta}$ vs rescaled time $t^{\prime}:=t / L^{z}$ for $r=3, b$ $=0.2$ and $\varepsilon=0.37323$ for $L=32,64,128,256$, and 512, showing a good data collapse. (c) Absorption time $\tau$ vs system size L, for $r$ $=3, b=-0.1$ and $\varepsilon=0.35200,0.35203,0.35205,0.35206$, and 0.35207 . Even small changes in $\varepsilon$ lead to substantial changes in the absorption time, and it is difficult to estimate the critical value of $\varepsilon$. (d) Rescaled average activity $m^{\prime}$ vs rescaled time $t^{\prime}$ for $r=3$, $b=-0.1$ and $\varepsilon=0.35203$ for $L=64,128,256,512$, and 1024, showing poor data collapse. Neither the initial decay nor the tails overlap; shown here is a compromise. Note that the initial decay is very slow, leading to a small estimate for the value of $\theta$.

exponent in the time direction $\nu_{\|}$and the correlation length exponent in space $\nu_{\perp}$. The scaling relation $\theta=-\beta / \nu_{\|}$connects the critical exponents.

To estimate the critical exponents for our CML, we performed direct numerical simulations and calculated the absorption time $\tau$ and the order parameter $m$, defined as the average activity. We used ensembles of initial conditions, in which all initial $u$ values are assigned a random value in the chaotic phase, $0 \leqslant u_{i}(0) \leqslant 1$. The $v$ values of the initial state are set to zero to ensure that they do not influence the $u$ values from the onset of iteration and that the analogy with the original model and our variant at $b=0$ is satisfied.

The behavior of the absorption time at criticality is used to determine the critical point and the $z$ exponent $[16,18]$. An ensemble of 128 systems is iterated forward in time until an absorbing configuration is reached. The average number of time steps needed before reaching such a configuration yields the absorption time $\tau$. Examples of $\tau$ as function of $L$ are shown in Figs. 5(a)-5(c); the best fit to a straight slope determines the critical exponent $z$.

In Figs. 5(b)-5(d) we plot examples of $m^{\prime}:=m L^{-\theta}$ as function of $t^{\prime}:=t / L^{z}$ for a range of $L \mathrm{~s}$. When proper scaling occurs, as is the case in Fig. 5(b), the curves for different $L$ fall on top of each other, and the initial power-law decay of $m^{\prime}$ determines the exponent $\theta$. Here an ensemble of 1000 systems was used. The order parameter was calculated as the sum of active sites divided by the total amount of sites. The systems are iterated forward until $t \approx L^{z}$, where the algebraic behavior clearly ends.

Estimates of critical exponents have been done for $r$
TABLE I. The critical exponents $z$ and $\theta=\beta / \nu_{\|}$for our CML. The values for DP are taken from Ref. [19].

\begin{tabular}{cllll}
\hline \hline$r$ & $b$ & \multicolumn{1}{c}{$\varepsilon_{c}$} & \multicolumn{1}{c}{$z$} & \multicolumn{1}{c}{$\theta$} \\
\hline 2.2 & -0.02 & 0.01338 & $1.57(1)$ & $0.16(1)$ \\
& -0.01 & 0.01465 & $1.57(1)$ & $0.16(1)$ \\
& 0.0 & $0.01605(2)$ & $1.53(1)$ & $0.17(1)$ \\
& 0.01 & $0.017628(5)$ & $1.57(1)$ & $0.16(1)$ \\
& 0.02 & $0.01921(2)$ & $1.57(2)$ & $0.17(2)$ \\
3.0 & -0.25 & $0.16312(3)$ & $1.58(1)$ & $0.160(5)$ \\
& -0.2 & $0.16495(2)$ & $1.58(2)$ & $0.168(3)$ \\
& -0.15 & $0.16205(1)$ & $1.58(1)$ & $0.17(1)$ \\
& -0.125 & $0.16368(2)$ & $1.57(1)$ & $0.20(1)$ \\
& -0.1 & $0.35203(1)$ & $1.52(3)$ & $0.02(2)$ \\
& 0.0 & $0.35984(3)$ & $1.42(2)$ & $0.18(1)$ \\
& 0.1 & $0.3393(1)$ & $1.48(2)$ & $0.155(1)$ \\
& 0.125 & $0.34745(5)$ & $1.53(2)$ & $0.15(1)$ \\
& 0.15 & $0.35680(5)$ & $1.57(1)$ & $0.159(3)$ \\
& 0.175 & $0.36545(1)$ & $1.58(1)$ & $0.16(1)$ \\
DP & 0.2 & $0.37323(1)$ & $1.58(1)$ & $0.16(1)$ \\
\hline \hline
\end{tabular}

$=2.2$ and $r=3.0$. For $r=3.0$, the critical exponents for the original model $(b=0)$ show significant deviations from the corresponding DP values and the computational costs are tolerable. In Figs. 5(a) and 5(b), we show examples of the rescaling plots for $r=3, b=0.2$, where a nice data collapse occurs and the transition appears to be of second order, and for $r=3, b=-0.1$ [Figs. 5(c) and 5(d)], where the data collapse is poor and the transition appears to be no longer continuous.

The values of the critical exponents are given in Table I and correspond simply to the best possible values, irrespective of the quality of the data collapse. For $r=2.2$, DP values are found for $|b| \geqslant 0.01$. For $r=3$, the critical transition on branch $B$ appear to be DP-like, while on branch $A$ a crossover to DP values is found when $b$ is large enough $(|b|>0.15)$. This regime coincides with values of $b$ where the solitons are suppressed in the space-time plots, and a continuous transition takes place. The soliton dominated dynamics at $b=-0.1$ is reflected in the extremely low value of the exponent $\theta$, characterizing the decay of the order parameter. Here the data collapse is rather poor as shown in Figs. 5(c) and 5(d).

\section{Spreading of turbulence in second-order regime}

So far the critical properties of the CMLs starting from "homogeneous" states have been studied, i.e., with initial conditions where each site in the lattice is assigned a random number in the chaotic (turbulent) phase. A different approach is to consider the spreading of a single turbulent seed in an otherwise laminar configuration (see Fig. 6). This makes it possible to study the dynamical critical exponents, or spreading exponents, and see how these compare to the directed percolation counterparts.

For spreading of activity in stochastic systems with absorbing states, the following quantities are characterized by 

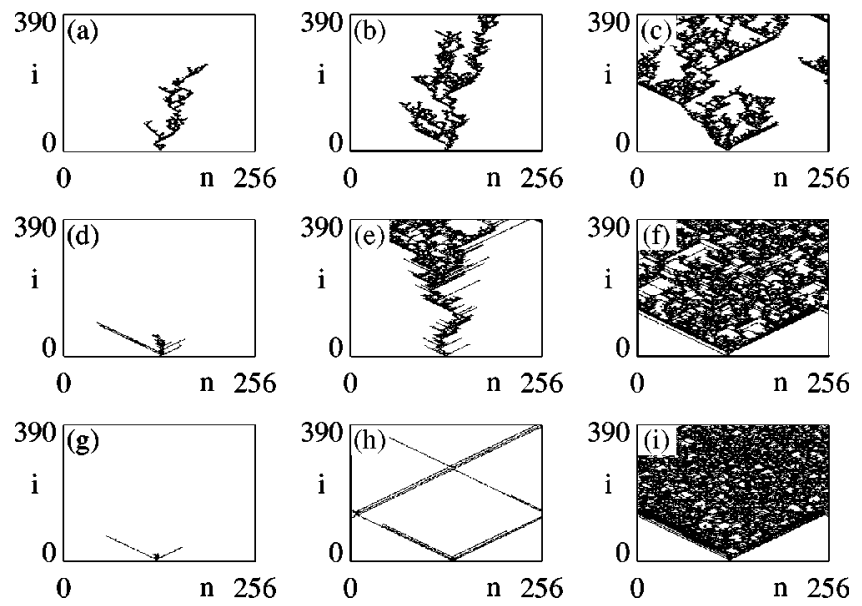

FIG. 6. Spreading of a single turbulent seed through the "natural-initial-state" below criticality, at criticality, and above criticality, for $b=0.2$ (a)-(c), the Chate-Manneville model for $b$ $=0$ (d) $-(\mathrm{f})$, and $b=-0.1(\mathrm{~g})-(\mathrm{i})$. In all cases $r=3.0$.

critical exponents [20]: the total number of chaotic sites $N(t)$, the survival probability $P(t)$, the mean-squared deviation $R^{2}(t)$ of the turbulent activity from the "seed," and the density $n(t)$ of chaotic sites within the spreading patch of turbulence. It is assumed that they behave according to

$$
N(t) \sim t^{\eta_{s}}, \quad P(t) \sim t^{-\delta}, \quad R^{2}(t) \sim t^{z_{s}}, \quad n(t) \sim t^{-\theta_{s}} .
$$

For probabilistic systems, it has been conjectured and verified numerically $[21,22]$ that the dynamical exponents satisfy the generalized hyperscaling relation $\eta_{s}+\delta+\theta_{s}=d z_{s} / 2$, where $d$ is the spatial dimension. For systems with a single absorbing state, including DP, one finds that $\delta=\theta_{s}=\beta / \nu_{\|}$ and $z_{s}=2 / z$, reducing the hyperscaling relation to $4 \delta+2 \eta_{s}$ $=d z_{s}$.

Systems with infinite numbers of absorbing states have been studied carefully recently and it has been found numerically that they differ from the classical ones with a single absorbing state by having what appears to be nonuniversal spreading exponents [22], which depend on into which absorbing state the spreading is taking place. Only exponents characterizing quantities averaged over surviving runs alone are found to be universal. It has thus been conjectured that $z_{s}$, the sum $\eta_{s}+\delta$, and $\theta_{s}$ are universal, whereas $\eta_{s}$ and $\delta$ individually are not. Only for the so-called "natural-initialstate" are the DP values found for the exponents characterizing quantities averaged over all runs. Such a particular state is constructed by letting the system evolve at criticality from homogeneous initial conditions, where all sites initially are in the active phase, until an absorbing configuration is reached. This scenario is rather unusual for critical phenomena and is still somewhat controversial, see, e.g., Ref. [23] for a different interpretation.

After a few spreading experiments in our CML, we indeed observed that the propagation of activity from the initial seed through the laminar region depended strongly on the configuration of the laminar state surrounding the seed. Moreover, the dynamical exponents varied with this configu-
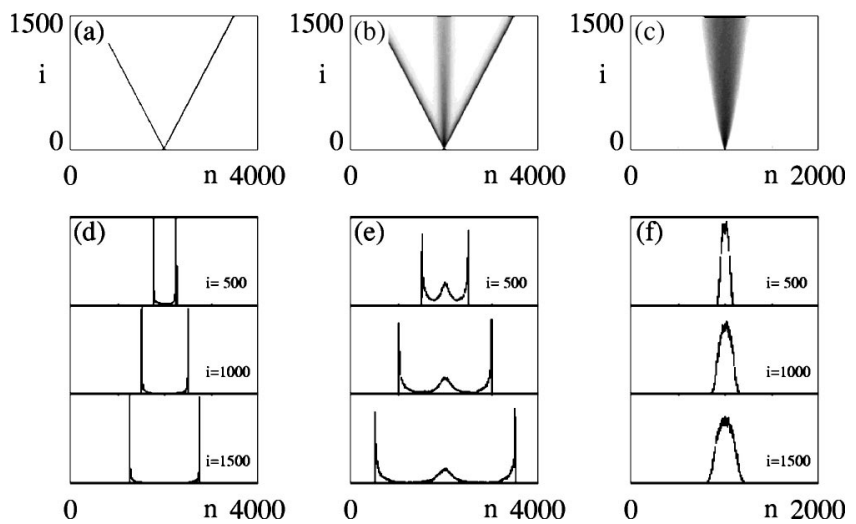

FIG. 7. The average spreading of active seeds in the naturalinitial-state close to criticality. The densities are obtained by averaging over $10^{4}$ realizations, and for clarity, we have included three snapshots of this average activity in the bottom rows. Parameters are $r=3$ (for all runs); and $b=-0.1[(\mathrm{a})$ and (d)]; $b=0.0[(\mathrm{~b})$ and (e)], and $b=0.2[$ (c) and (f)]. The respective values of naturalinitial-state are 1.170, 1.212, and 1.235.

ration, thus being nonuniversal. So the nonunique absorbing state of our CML (any configuration with all $u$ values above unity and $v$ values not too large will be absorbing) leads to behavior as can be expected for DP with an infinite numbers of absorbing states.

We determined the natural-initial-state by iterating systems of up to 4096 sites from homogeneous initial conditions until an absorbing configuration is reached. The average value of all sites is then used as the value of the laminar background. For $r=3.0$, we have calculated these as 1.235 for $b=0.2,1.212$ for $b=0,1.170$ for $b=-0.1$, and 1.0395 for $b=-0.2$.

In Fig. 7, we display the average spreading for $r=3$ and $b=-0.1,0$, and 0.2. Clearly, for $b=-0.1$ and for the Chaté-Manneville model at $r=3, b=0$ it is basically impossible to estimate the spreading exponents at the naturalinitial-state, since the spreading is dominated by the solitons [see Figs. 7(b) and 7(e)]. This behavior is distinctly different from what is observed in the various systems belonging to the DP universality class. We have, therefore, only estimated the spreading exponents for $b=0.2$ at branch $A$, and $b=$ -0.2 at branch $B$; in both cases the solitons are not dominant.

Note that the strength of the spreading solitons can be altered by changing the value of the laminar background. By
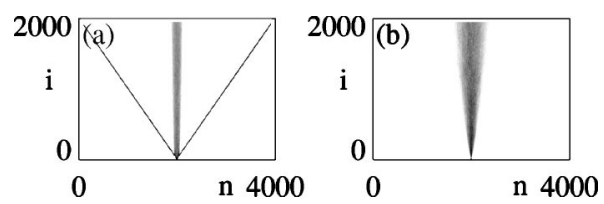

FIG. 8. Average spreading activities near criticality for $r=3, b$ $=0.2$ (a) and $r=3, b=0$ (b). In comparison to the spreading into the natural-initial state as shown in Fig. 6, the value of $u$ in the laminar background has been lowered from 1.235 to 1.225 in (a), thus strongly enhancing solitons, and has been increased from 1.212 to 1.22 in (b), thus strongly suppressing soliton activity. 


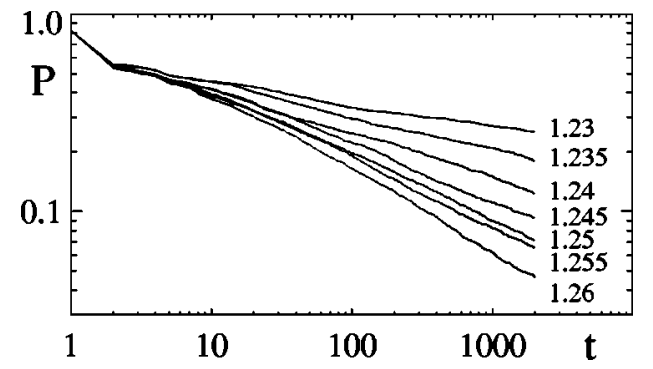

FIG. 9. Average survival probabilities for spreading clusters in our CML with $r=3$ and $b=0.2$ for various values of the background (indicated in the figure). The natural background state has $u=1.235$.

increasing the background to a value above the naturalinitial-state one, the solitons can be suppressed, while they can be enhanced by a decrease of the laminar value; see Fig. 8.

Our estimates of the dynamical exponents have been done for simulations with a maximum time of 2000 iterations. An active seed is placed in the center of the lattice, surrounded by a laminar background. The seed consists of two active sites, each of which is assigned a random number in the chaotic regime, such that the location is fixed but the values of the active sites differ for each trial in the ensemble. The ensemble size $N_{s}$ used for statistical averaging and the number of sites in the lattice $L$ have been adjusted to the number of surviving runs the different setups produced, and how far the turbulence propagated out from the seed. A minimum of 200 surviving runs have been used in the averaging. In Fig. 9, we show a typical example of the curves that have been

TABLE II. Estimated spreading exponents for $r=3.0$ for background values $x_{i}$. The deviation from the hyperscaling relation for $d=1$ is defined as $\Delta \equiv z_{s} / 2-\left(\eta_{s}+\delta+\theta_{s}\right)$. Note that for $b=0.2$, the natural background state has $x_{i} \approx 1.235$; for this value, the exponents $\delta$ and $\eta_{s}$ are close to their DP values. Similarly for $b$ $=-0.2$, the natural background state has $x_{i} \approx 1.1395$; again $\delta$ and $\eta_{s}$ are close to their DP values.

\begin{tabular}{rlrcrrr}
\hline \hline$b$ & \multicolumn{1}{c}{$x_{i}$} & \multicolumn{1}{c}{$z_{s}$} & \multicolumn{1}{c}{$\delta$} & \multicolumn{1}{c}{$\eta_{s}$} & \multicolumn{1}{c}{$\theta_{s}$} & \multicolumn{1}{c}{$\Delta$} \\
\hline 0.2 & 1.229 & $1.98(2)$ & $0.00(0)$ & & & \\
& 1.23 & $1.68(2)$ & $0.10(1)$ & $0.43(2)$ & $0.32(1)$ & $-0.01(2)$ \\
& 1.235 & $1.60(1)$ & $0.16(1)$ & $0.34(1)$ & $0.29(1)$ & $0.01(1)$ \\
& 1.24 & $1.61(2)$ & $0.23(2)$ & $0.25(1)$ & $0.31(1)$ & $0.01(2)$ \\
& 1.245 & $1.65(1)$ & $0.30(1)$ & $0.23(1)$ & $0.29(2)$ & $0.00(1)$ \\
& 1.25 & $1.65(3)$ & $0.34(2)$ & $0.14(1)$ & $0.300(3)$ & $0.05(2)$ \\
& 1.255 & $1.69(2)$ & $0.35(2)$ & $0.14(2)$ & $0.29(1)$ & $0.07(2)$ \\
& 1.26 & $1.72(3)$ & $0.43(1)$ & $0.04(1)$ & $0.29(1)$ & $0.10(2)$ \\
0.2 & 1.13 & $1.99(1)$ & $0.00(0)$ & $0.793(2)$ & $0.205(1)$ & $0.00(1)$ \\
& 1.135 & $1.59(2)$ & $0.09(1)$ & $0.42(1)$ & $0.29(1)$ & $-0.01(1)$ \\
& 1.139 & $1.58(2)$ & $0.170(3)$ & $0.32(1)$ & $0.30(1)$ & $0.00(1)$ \\
& 1.1395 & $1.58(1)$ & $0.16(1)$ & $0.31(1)$ & $0.28(1)$ & $0.04(1)$ \\
& 1.145 & $1.56(1)$ & $0.249(2)$ & $0.20(1)$ & $0.24(2)$ & $0.08(2)$ \\
& 1.15 & $1.61(1)$ & $0.347(3)$ & $0.11(1)$ & $0.27(1)$ & $0.08(1)$ \\
& 1.155 & $1.68(3)$ & $0.45(1)$ & $0.01(1)$ & $0.22(1)$ & $0.16(1)$ \\
DP & & 1.26523 & 0.15947 & 0.31368 & 0.15947 & \\
\hline \hline
\end{tabular}

used to extract the exponents in Table II. The figure shows the survival probability for various choices of background and thus gives the exponent $\delta$. Whether these curves display true power-law behavior (even away from the natural background state) is hard to judge, but we believe that it is at least a reasonable interpretation [24].

Our results in Table II agree rather well with previously obtained results for probabilistic systems with an infinite number of absorbing states. In particular, the exponents averaged over surviving runs alone definitely seem to be universal as long as the background does not deviate too much from the natural-initial-state one. While the values for the sum $\delta+\eta_{s}$ are very close to the DP value of $0.47315(7)$, our results for $z_{s}$ and $\theta_{s}$ deviate from their respective DP values. A very interesting observation is that the hyperscaling relation is satisfied ( $\Delta \simeq 0$ ) for the majority of different background values. Only for the highest values are significant deviations encountered.

\section{STOCHASTIC MODEL}

The propagating structures, which are observed in the CML that we studied in the preceding section, appear to play an important role for the transitional behavior. It is, however, numerically very demanding to obtain good statistics for large CMLs and long times. As pointed out already in the introduction, this is the reason why one tries to map such deterministic models to simple stochastic models. Not only may there be more hope to understand such models analytically, they also are much easier to handle from a computational point of view.

In this section, we will introduce and study a very simple extension of the Domany-Kinzel cellular automaton that itself is a simple model showing DP behavior. While for the Domany-Kinzel automaton, every site can only be active or inactive, we will allow sites to either contain a left or right traveling soliton. As in the CML, these solitons should be generated from active sites only, and we wish to be able to tune their typical lifetime. The only process in which these solitons aid the spreading of activity is by collisions: for simplicity, we assume that with probability 1 , a pair of colliding solitons yields a single active site.

Below we will first discuss the definition of our model in Sec. III A. We will then discuss the mean-field equations for our model in Sec. III B, and these will show a transition from second- to first-order behavior. We will study the statistical properties of our model in Sec. III C. We will illustrate the role of solitons in direct simulations of this model; these simulations will point to the relevance of large "holes" that cannot be "healed" by the solitons. We will discuss the statistical properties of our model near the transition from inactive to active states in the soliton-dominated parameter regime. We will find that the transition is no longer in the DP universality class, since no asymptotic scaling regime can be reached. While the transition shows some characteristics of a first-order transition (dependence on initial state, for example), the asymptotic situation is not entirely clear: rather we find a regime of long lived transient states between active and inactive regimes. 


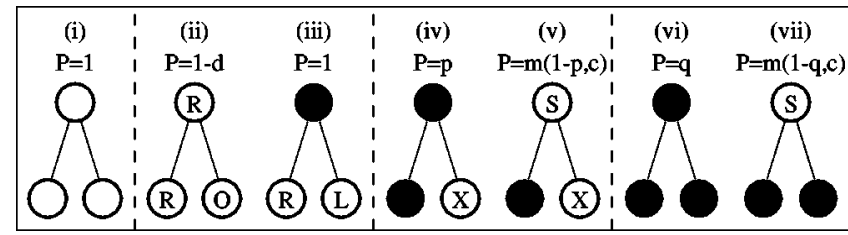

FIG. 10. Schematic definition of our stochastic model. The bottom two circles denote possible incoming states and the circle at top denotes the possible stochastic outcome. Our model is defined on a diamond lattice and so one only needs to define the probabilities for certain offspring (active, inactive, right or left traveling soliton) as a function of its two predecessors. Empty circles depict inactive states, black circles are active, " $R$ " and " $L$ " denote right- and left-moving solitons, " $S$ " denotes a soliton of arbitrary direction, and " $X$ " finally represents any state $(L, R$, inactive or active). The notation $m(a, b)$ denotes the minimal value of $a$ and $b$, and $P$ denotes the conditional probability that this outcoming state occurs. See text for a more elaborate explanation.

\section{A. Definition of model}

The $(1+1)$-dimensional Domany-Kinzel cellular automaton is defined on a diagonal square lattice, where each site can either be active or inactive. The model evolves by parallel updates according to the transition probabilities $p$ and $q$, corresponding to the probabilities that an empty plus an active site or two active sites, respectively, produce a single active site. The choice $q=p(2-p)$ corresponds to a realization of directed bond percolation [1].

In our extension the active sites behave like usual directed bond percolation except from the fact that with probability $c$ they can emit a left- or right-moving soliton. These solitons have a tunable lifetime and travel ballistically. We assume that the solitons cannot, by themselves, create chaos, except when two solitons collide.

The updating rules illustrated in Fig. 10, where the sites can be either inactive (empty), active (black), or contain a left- or a right-moving soliton, are as follows.

(i) The inactive state: two inactive sites always yield an inactive site. This property ensures that there is a unique absorbing state.

(ii) Soliton propagation: a right-moving soliton either dies with probability $d$, or propagates with probability $(1-d)$, when the "O" state to its right- is inactive or another rightmoving soliton. The rule for left-moving solitons follows by left-right symmetry.

(iii) Soliton collision: when two oppositely propagating solitons collide, they generate an active site with probability one. This is the only process where solitons lead to spread of active sites. In principle, we could generate active sites with a probability less than one, but it may be expected that this does not change the behavior of the model in a qualitative sense.

(iv) Single active sites: a single active site, where $X$ can either be a soliton or inactive site, leads with probability $p$ to a new active site. Note that the spreading of activity is thus not enhanced by individual solitons enhanced by individual solitons. (v) Transformation: a single active site can give rise to a soliton $(\mathrm{S})$ with probability $\min (1-p, c) ; c$ denotes the creation rate of solitons. Such a new soliton can be either leftor right-moving with equal probability.

(vi) Pair of active sites: two active sites create a new particle with probability $q$; we restrict ourselves to bonddirected percolation and take $q=p(2-p)$.

(vii) Soliton creation from pair of active states: similar to case (v), a pair of active sites can give rise to solitons with probability $\min (1-q, c)$.

\section{B. Mean-field equations}

To interpret the physical properties of our cellular automaton, a crude insight can be obtained by applying meanfield theory. In this approximation, it is reasonable to ignore the differences between left and right traveling solitons, and so our mean-field equations are for two concentrations, those of chaotic sites $c$ and solitons $s$.

(a) Equation for chaotic sites. Chaotic sites can emit solitons and can be generated by collisions of two solitons; apart from these two rules they behave like DP. Thus, without the solitons, the rate equation (without noise) would be $\dot{c}=b_{1} c$ $-b_{3} c^{2}[13]$. To incorporate the creation of an active site, when two solitons collide according to rule (iii), the term $b_{2} s^{2}$ needs to be added to this equation. There is no source term linear in $s$ in the rate equation for $c$, reflecting that we assume that individual solitons do not give rise to activity. Note that, for simplicity, we have not distinguished between right- and left-moving solitons

(b) Equation for solitons. There are four processes that influence the solitons. Solitons may decay spontaneously according to rule (ii), and this yields a term $-a_{3} s$ in the rate equation for $s$. Solitons also die upon collision leading to a term $\propto-s^{2}$. Depending on the lifetime of the solitons, either of these two terms may dominate and so we keep both of them; we will see below that this will indeed be a crucial ingredient. Solitons are created from active sites according to rule (v) and (vii). While this in principle yields source terms in the rate equation of $s$ proportional to both $c$ and $c^{2}$, we only keep the linear term, since the prefactor for both these terms will be of the same order. Inclusion of the quadratic term does not affect the qualitative dynamics.

The rate equation for the solitons and chaotic sites can then be written as

$$
\begin{aligned}
& \dot{s}=a_{1} c-a_{2} s^{2}-a_{3} s, \\
& \dot{c}=b_{1} c+b_{2} s^{2}-b_{3} c^{2},
\end{aligned}
$$

where the lifetime of the solitons is set by $1 / a_{3}$ and the spreading rate of the chaotic patches by $b_{1}$.

These two equations can be simplified by the introduction of a rescaled of time $\tau$ and densities $S$ and $C$ to be

$$
\begin{gathered}
\dot{S}=C-S^{2}-a S, \\
\dot{C}=r_{0} C+S^{2}-u C^{2},
\end{gathered}
$$




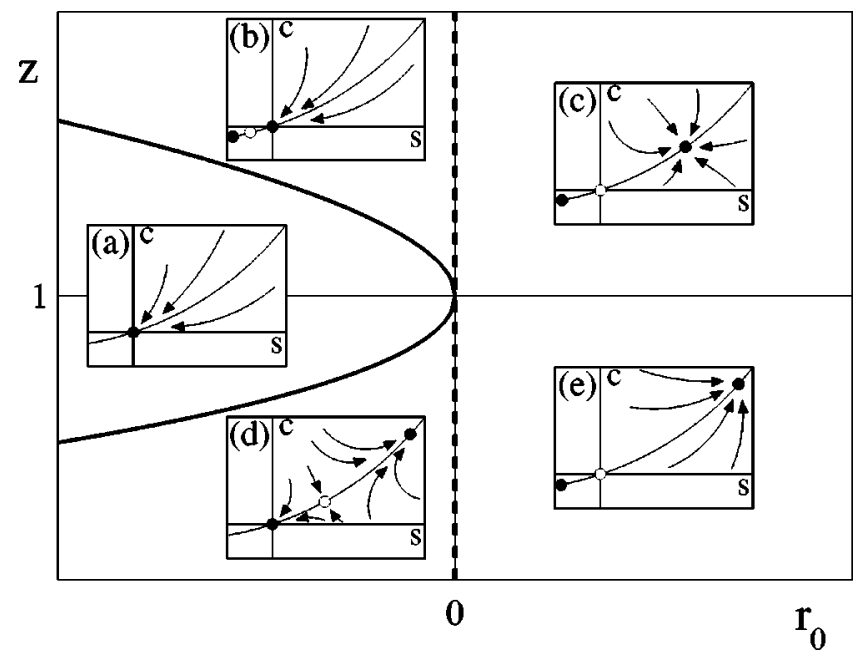

FIG. 11. Dynamical system analysis of the mean field equations (10) and (11). The full and dashed curves show the location in $r, z$ space of the saddle-node and transcritical bifurcations, respectively. The five insets (a)-(e) show schematically the flow in the various regimes of the mean field equations. For more details see text.

where

$$
\begin{gathered}
a=\frac{a_{2} a_{3}}{a_{1} b_{2}}, \quad r_{0}=\frac{a_{2} b_{1}}{a_{1} b_{2}}, \quad u=\frac{b_{2} b_{3}}{a_{2}^{2}} \\
t=\frac{a_{2}}{a_{1} b_{2}} \tau, \quad s=\frac{a_{1} b_{2}}{a_{2}^{2}} S, \quad c=\frac{a_{1} b_{2}^{2}}{a_{2}^{3}} C .
\end{gathered}
$$

We will now analyze the possible transitions in the meanfield equations (12) and (13).

(c) Fixed points. The fixed points $\left(S^{*}, C^{*}\right)$ of the rescaled equations (10) and (11) satisfy $C^{*}=S^{* 2}+a S^{*}$, where $S^{*}$ is given by solutions to the fixed point equation

$$
\begin{gathered}
S f(S)=0, \\
f(S)=u S^{3}+2 u a S^{2}+\left(u a^{2}-1-r\right) S-a r_{0} .
\end{gathered}
$$

Apart from the trivial fixed point $\left(S^{*}, C^{*}\right)=(0,0)$, there may be either 1 or 3 other fixed points that can be found from solving Eq. (15). It can be shown that Eq. (15) always has one solution for large negative $S$. This fixed point can be ignored since only points where both $S^{*}$ and $C^{*}$ are positive are relevant for our mean-field equations (remember that $S$ and $C$ are both concentrations). The two nontrivial fixed points $\left(S_{1}^{*}, C_{1}^{*}\right)$ and $\left(S_{2}^{*}, C_{2}^{*}\right)$ are born in a saddle-node bifurcation, when the discriminant of Eq. (15) becomes negative. Introducing the parameter $z:=a^{2} u$ and performing the tedious standard algebra, yields that this occurs when

$$
z^{2}-\left(2-5 r_{0}-r_{0}^{2} / 4\right)+\left(1+r_{0}\right)^{3}=0
$$

and so the locus of the saddle-node bifurcation only depends on $r_{0}$ and $z$. It can also be shown that at $r_{0}=0$, always one of the nontrivial fixed points crosses through the fixed point of the origin in a transcritical bifurcation. The various types of flows that occur as function of $z$ and $r_{0}$ are illustrated in Fig. 11.

As shown in Fig. 11, there are essentially four qualitatively different types of flow and two bifurcations occurring. We will here discuss these flowtypes and their relevance for the dynamics as follows.

(a) Only the trivial fixed point is present, and is stable. Hence all initial conditions flow to the absorbing state.

(b) For small soliton lifetime $z>1$, the two nontrivial fixed points $\left(S_{1}^{*}, C_{1}^{*}\right)$ and $\left(S_{2}^{*}, C_{2}^{*}\right)$ that are born in a saddlenode bifurcation do not lie in the first quadrant and are, therefore, not relevant for the mean-field equations. Hence, this situation means that there is a single relevant fixed point at the origin and so the system is in the absorbing state.

(c) When, for $z>1, r_{0}$ crosses through zero from below, $\left(S_{1}^{*}, C_{1}^{*}\right)$ crosses through the origin in a transcritical bifurcation. All initial conditions in the first-quadrant flow now to $\left(S_{1}^{*}, C_{1}^{*}\right)$; the mean-field equations indicate that there is a finite activity, whose value grows approximately linearly in $r_{0}$. The transition at $r_{0}=0$ corresponds to the standard DP transition for $z>1$.

(d) For long soliton lifetimes $(z<1)$, the two nontrivial fixed points $\left(S_{1}^{*}, C_{1}^{*}\right)$ (square) and $\left(S_{2}^{*}, C_{2}^{*}\right)$ (triangle) are also created in a saddle-node bifurcation; but in contrast to case (b) both lie in the first quadrant and are, therefore, relevant for the dynamics. Depending on initial conditions, the final state can either be absorbing or active; the incoming manifold of the saddle point acts as a separatrix. The transition that occurs here as the saddle-node bifurcation is crossed leads to a finite jump in the value of $c$ in the active state, which is indicative of a first-order transition.

(e) When, for $z<1, r_{0}$ crosses through zero from below, $\left(S_{2}^{*}, C_{2}^{*}\right)$ crosses through the origin in a transcritical bifurcation. All initial conditions in the first quadrant flow now to $\left(S_{2}^{*}, C_{2}^{*}\right)$.

To study the phase transition, we shall primarily vary $r_{0}$ while keeping $a$ and $u$ fixed. There are following three generic choices for $z$ relevant here.

(i) $z \rightarrow \infty:$ In this case the solitons have probability 1 to die once they are generated, and so the system is effectively soliton free. This is the case of pure DP, and the transition takes place at $r_{0}=0$. There is no hysteresis.

(ii) $z>1$ : This is the regime of short soliton lifetimes. Here the solitons do not contribute to any change in the qualitative behavior. An attractive fixed point $S=S_{1}^{*}$ $\approx a r /(z-1)$ emerges for small, positive $r_{0}$. This corresponds to $C=C_{1}^{*} \approx a^{2} r_{0} /(z-1)$, such that this fixed point converges towards the DP value $C_{1}^{*} \rightarrow r_{0} / u$ for large $a$. As $r_{0} \rightarrow 0$, this fixed point converges towards the origin and it changes stability at $r_{0}=0$ (see Fig. 11), implying that the transition is continuous. Thus, the transition for small soliton lifetimes $(z>1)$ still takes place at $r_{0}=0$ and resembles DP.

(iii) $z<1$ : This is the soliton dominated regime, where a completely different scenario occurs. For $r_{0}>0$, the behavior is determined by the stable node at $S_{2}^{*} \approx a\left(z^{-1 / 2}-1\right)$. When $r_{0}$ becomes negative, this fixed point remains stable and away from the origin. Simultaneously the origin be- 

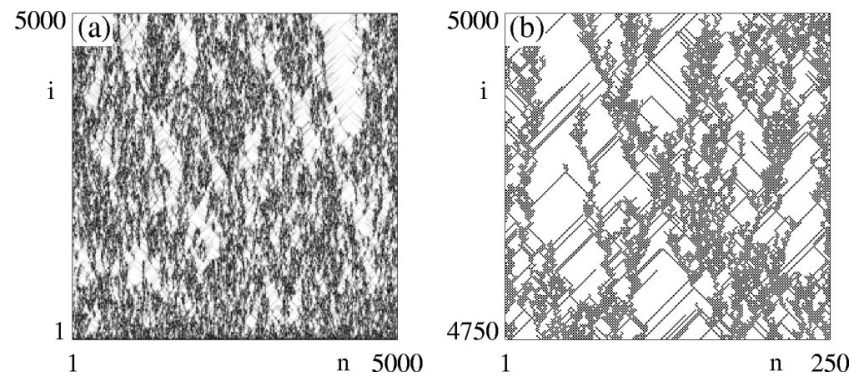

FIG. 12. (a) Large scale dynamics of our stochastic model for $d=0.01, c=0.1$, and $p=0.614$. The gray scale corresponds to the number of solitons and active sites coarse grained in a cell of 20 space and 20 time units. (b) Enlargement of the dynamics shown in the top left corner of (a).

comes attractive and a saddle appears close to the origin at $S=S_{1}^{*} \approx a r_{0} /(z-1)$. Initial conditions close to the origin will evolve into that point, while initial conditions above the stable manifold for the saddle located at $\left(S_{1}^{*}, C_{1}^{*}\right)$ will converge toward the node $\left(S_{2}^{*}, C_{2}^{*}\right)$. This will go on until the saddle $\left(S_{1}^{*}, C_{1}^{*}\right)$ and the node $\left(S_{2}^{*}, C_{2}^{*}\right)$ merge in a saddlenode bifurcation at $r_{0}=r_{c}(z)$. Below this critical point, the origin is globally attractive and every trajectory in the phase space converges towards this. Going back and forth along scenarios (a), (d), and (e) there is hysteresis and so for $z$ $<1$, we clearly observe a first-order transition.

For infinite soliton lifetimes $(a=0, z=0)$, the critical point is shifted down to $r_{0}=-1$. Setting $a=0$ into Eq. (15) yields the fixed point $S^{*}=\sqrt{\left(1+r_{0}\right) / u}$ that shows that the transition is continuous, but with $\beta=1 / 2$ instead of the DP mean-field value, $\beta_{D P}=1$.

Finally, at the tricritical point $z=1$, Eq. (15) is reduced to $a^{2} f(S)=S^{3}+2 a S^{2}-a^{2} r_{0} S-a^{3} r_{0}$. At $r_{0}=0$, the only nonnegative root is $S=0$, but for small positive $r_{0}$ a new root appears at $S^{*} \approx a \sqrt{r_{0} / 2}$. The transition thus remains at $r_{0}$ $=0$ and is continuous, but again with $\beta=1 / 2$ instead of the DP value $\beta_{D P}=1$.

\section{Phenomenology and statistical properties of the stochastic model}

Let us now discuss the properties of the full stochastic model based on direct numerical simulations. For small but finite values of the soliton lifetime $(d \gg 0)$ or for sufficiently small production of solitons $(c \ll 1)$, the transition from inactive to active states that occurs when $p$ is increased is of second order and indeed appears to be in the DP universality class. There is, however, also a regime in which the model appears to display a first-order transition. In the remainder of the discussion on the stochastic model, we will focus on this regime, which shows some interesting new features.

(a) Phenomenology. The phenomenology of this regime will be illustrated following Figs. 12 and 13, where different aspects of the dynamics of our model are shown. The parameters chosen are somewhere in the transitory regime, which in the mean-field description corresponds to the regime with two stable fixed points [Fig. 11(b)].

In Fig. 12(a), we show the evolution of our model, starting from a fully active state. Figure 12 (b) is a closeup of the
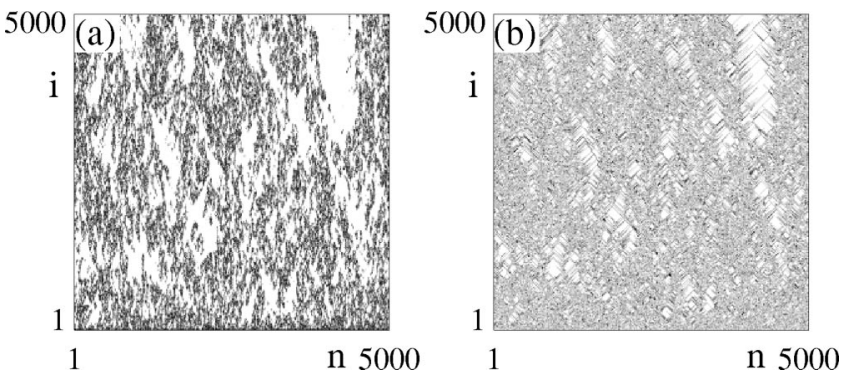

FIG. 13. Concentration of active sites (a) and solitons (b) for the state shown in Fig. 12.

top left corner of Fig. 12(a) which shows the dynamics of active sites and solitons in detail. At first glance, the clusters of activity look extremely similar to the ordinary DomanyKinzel Cellular Automaton, but after closer inspection it becomes clear that colliding solitons generate new active clusters [one example can be seen in Fig. 12(b) for $n \approx 50$, $i \approx 4825]$. We have shown the coarse grained activity and solitons separately in Figs. 13(a) and 13(b). Clearly, the soliton density is more uniformly spread, and one can think of the coarse grained dynamics as active clusters surrounded by clouds of solitons.

(b) Decay of activity. To gain insight in the statistical properties of our model, we have studied the decay of the number of active sites as a function of time, for a range of system sizes $L$ and parameter values $p$. Unless noted otherwise, we keep the soliton parameters $d$ and $c$ at values 0.01 , and 0.1 respectively. In Fig. 14, we show the results of these calculations for $p$ ranging from 0.612 to 0.621 .

For early times $\left(t<10^{3}\right)$, one could misinterpret that data as being indicative of a second-order transition with non-DP exponents. When $p$ is small enough $(<0.61)$, the activity decays faster than a power law, while when $p$ is large

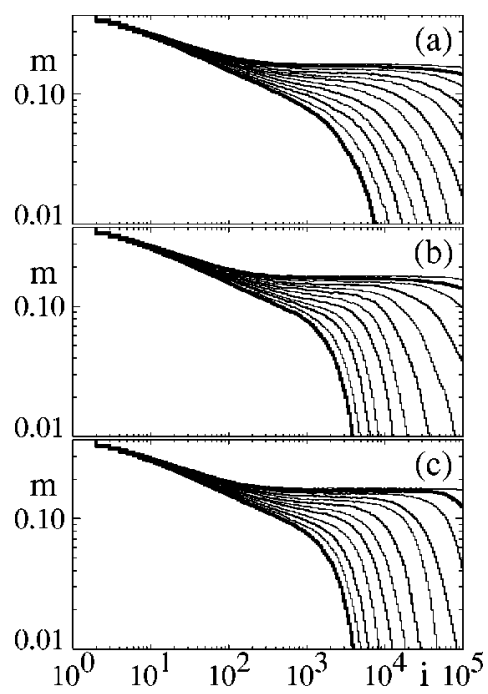

FIG. 14. Decay of average activity $m$ for $c=0.1, d=0.01$, and $p=0.610,0.611, \ldots, 0.621$ (increasing $p$ leads to an increase of activity; the curves with $p=0.610$ and 0.620 are thicker). Averages are taken over (a) 2000 systems of $\mathrm{L}=200$, (b) 200 systems of $\mathrm{L}$ $=2000$, and (c) 20 systems of $L=20000$. 


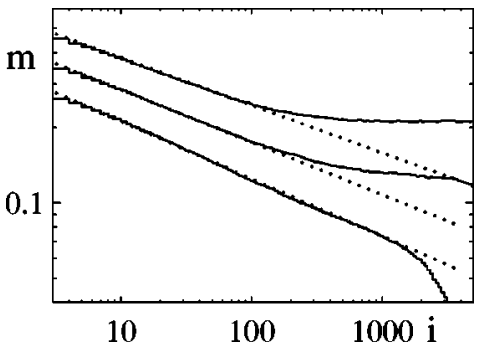

FIG. 15. Average activity $m$ for $c=0.1, d=0.01$ in an ensemble of 20 systems of $L=20000$, showing the appearance of quasipower-law decay. The three curves correspond to $p=0.612,0.616$, and $p=0.62$, respectively, and are shifted by $30 \%$ for clarity. The three straight lines corresponds to power laws with exponents $-0.19,-0.21$, and -0.23 . In particular, the scaling in the system for $p=0.612$ looks rather convincing with an exponent of -0.23 .

enough, the active state does not decay, and we are above the transition and the activity reaches a plateau value. For DP, there is a critical value of $p$ where the activity decays as a power law. As shown in Fig. 15, our model displays a transient decay towards the plateau value which can look like a power law. For a transient period that in this case goes up to $t \approx 10^{3}$, it is possible to find values of $p$ such that the decay of $m$ appears to be a power law with a non-DP exponent. We speculate that this transient behavior may be the origin of some of the nonuniversal power laws observed in coupled map lattices $[5,7,18,25]$, where it is very hard to reach (effective) large times.

For this "scaling" to be truly asymptotic, one should be able to extend the scaling regime to arbitrary large times; however the activity curves for system sizes 200, 2000, and 20000 all bend downwards at nearly the same time; hence, there is no hope that increasing the systemsize extends the time interval over which apparent scaling can be found. For times longer than $10^{3}-10^{4}$, the activity either decays rapidly, or first hits a plateau. Clearly, the transition in our model is not an ordinary second-order transition. If we focus on the activity as a function of $p$ for a fixed large time $t>10^{4}$, we find a very abrupt transition from an inactive to an active state, with a value of the activity given by the "plateau" that can be seen in Fig. 14. This behavior is indicative of a firstorder transition, consistent with the mean-field theory for large soliton lifetimes.

(c) Nucleation of holes. Is this transition now an ordinary first-order transition, and if so, what would be the critical value of $p$ ? From the magnetization curves, such as shown in Fig. 14, it is not so easy to answer this question; in particular, we observe that the plateau is not the truly asymptotic state for these parameters, since decay eventually sets in. We will now first study the reason for this decay. Let us return to Fig. 13, where the activity appears to arrive at the plateau (the overall activity appears to approach a constant). However, around $n \approx 4000$ and $i \approx 3000$ a large "hole" opens up. Once the size of this hole becomes larger than twice the lifetime of the solitons, it becomes unlikely that colliding solitons will create new activity there and "heal" the hole. In fact, for this particular example, the hole did spread out and the system decayed to the inactive state. A closer inspection of the
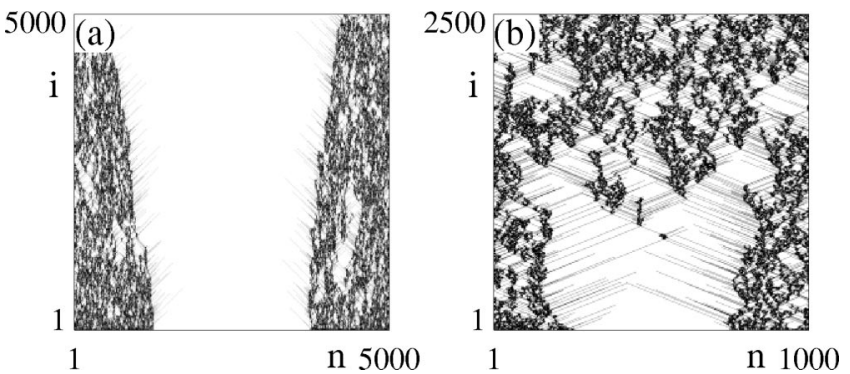

FIG. 16. Dynamics for $c=0.1, d=0.01$, and $p=0.618$, showing that a large hole of size 2500 is not healed (a), while a hole of size 500 is healed (b).

dynamical states that occur when the activity drops below the plateau value, shows that this is the general scenario: the nucleation and subsequent spreading of a large inactive droplet is what dominates the asymptotic decay of the active states here.

We illustrate this property by following the dynamics of a large inactive droplet for $p=0.618$, where the system has a well-defined plateau in the activity (see Fig. 14). A hole of size 2500 grows as can be seen in Fig. 16(a), while a hole of size 500 is healed for these same parameter values [Fig. 16(b)]. The difference between the spreading of a small active cluster and the behavior of an homogeneously active state indicates that the initial concentration of active sites plays a role. This is illustrated in Fig. 17, where we follow the evolution of the activity for a range of initial concentrations of activity for $p=0.621$. For initial activities in the range from 1 to 0.1 , the same plateau value is reached, but for initial activities of 0.05 and smaller, there is an initial increase of the activity after which the activity rapidly decays; the plateau is never reached.

Finally, in Fig. 18, we show the evolution of the activity $m$ divided by the number of surviving clusters for the same parameter values. For small systems, these plots are very different from the ones averaged over all systems (Fig. 14); in the present case there is a typical activity in each system which rapidly disappears. We interpret this as further evidence that the nucleation of large holes dominates the eventual decay. For larger systems, this effect disappears because

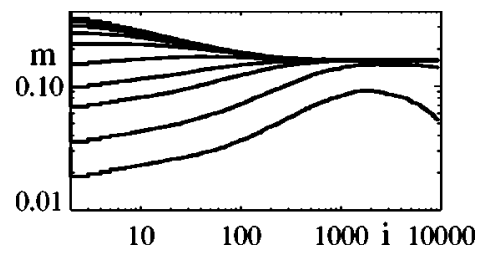

FIG. 17. Evolution of the average activity $m$ in 20 systems of size 20000 for $c=0.1, d=0.01$, and $p=0.621$, i.e., well in plateau regime. Here we vary the initial concentration of active states by randomly distributing active sites through our lattice for $i=1$. These activities are, respectively, $1.0,0.85,0.7,0.55,0.4,0.25$, $0.15,0.1,0.05$, and 0.025 . For the latter two cases, the plateau is not reached, even though initially the activity is increasing. That the long-time behavior $(i>200)$ depends on the initial concentration is reminiscent of a first-order transition. 


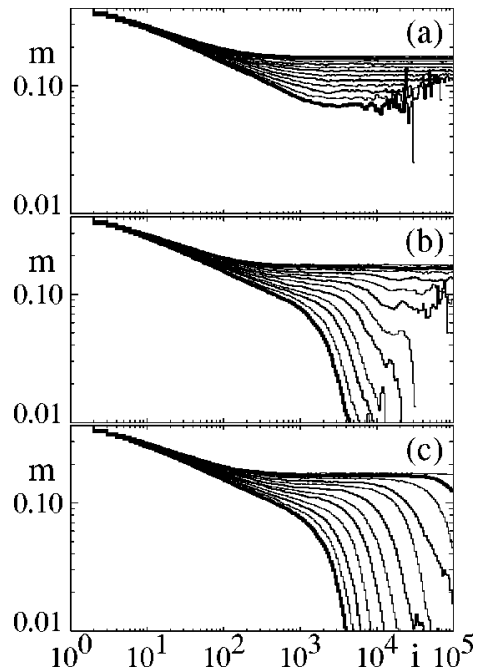

FIG. 18. Average activity divided by the number of active systems, for the same parameter values as shown in Fig. 14.

the time it takes for a hole to engulf the whole system is large.

(d) Nature of the transition. Purely from the activity curves such as shown in Fig. 14, it is very difficult to determine the transition value $p_{c}$. For very large holes, or equivalently a large droplet in an infinite inactive background, one can however determine the propagation velocity of the "domain wall" that separates the active from the inactive state. In the examples shown above, such an active droplet would shrink, and the "plateau" is not the asymptotic state. One could imagine that the real transition occurs when active droplets start to spread. Even the nucleation of a large hole will then not destroy the active state.

We have performed simulations of the shrinking and spreading of active patches of size 5000 in an inactive background (see Fig. 19), from which we conclude that the active state starts to spread into the inactive state for $p>p^{*}$ $\approx 0.63012(4)$. Note that Fig. 14 indicates that for such value, we are already deep into the plateau regime.

In addition, we did some simulations to determine the critical value of $p$ where the domain wall velocity changes sign as a function of $d$, the inverse soliton lifetime. These simulations indicate that for $d \searrow 0$, i.e., for infinite soliton times, there is a well-defined domain wall velocity that changes sign for $p \approx 0.6298(2)$; furthermore, $p^{*}(d)$ appears to be a smooth function [see Fig. 19(b)].

We conclude from this that the best way to find the transition point is to study the spread of a domain wall between an active and inactive state. To determine the nature of the transition, one needs to inspect activity graphs like Fig. 14. When, for large systems, there is no plateau, the transition is of second order, while for cases such as presented in Fig. 14, the transition is most likely of first order. The lifetime of the solitons introduces a much larger crossover time for the nucleation of sufficiently large holes. This leads to a range of $p$ values where an isolated droplet does not spread, or equivalently, a large enough hole does not heal, but nevertheless a very long lived transient first-order-like plateau state is reached.
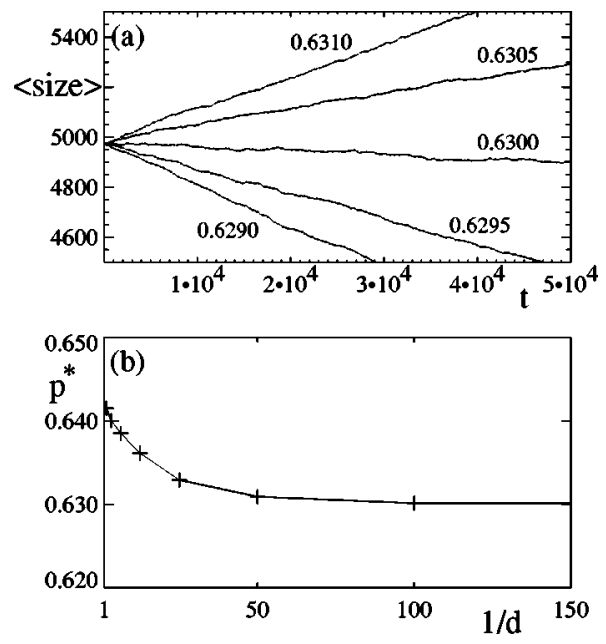

FIG. 19. (a) Time evolution of the average size of an active droplet (averaged over 500 realizations) of initial size 5000, for $p$ $=0.631,0.6305,0.063,0.6295$, and 0.629 . The slope of these curves varies linearly with $p$ in this regime, yielding that the droplet starts to spread for $p=0.63012(4)$. (b) Critical value of $p, p^{*}$, where front velocity changes direction as a function of the soliton lifetime $1 / d$, for $c=0.1$. The error bars are smaller than the symbol size. For $d \searrow 0, p^{*} \searrow 0.6928(2)$, while for $d=1, p^{*}$ approaches $0.6415(5)$. This latter value is slightly different from the soliton-free value; even though $d=1$, solitons life for one time step and hence slightly alter the critical value.

\section{DISCUSSION}

The overall picture that emerges from our study is that the transition to spatiotemporal intermittency is strongly influenced by coherent ballistically traveling "solitons," which, even though they have a finite lifetime, change the nature of the transition and can introduce first-order-like behavior. That such a scenario is relevant, is supported by recent evidence for a discontinuous transition to spatiotemporal chaos in the damped Kuramoto-Sivashinsky equation [26], which is well known to support localized ballistically moving excitations, or "pulses" [27].

We build our conclusions upon an extension, using twodimensional local maps, of the Chate-Manneville coupled map lattice. We thereby gain an additional parameter, which turns out to tune the importance and lifetime of the solitons. For this coupled map lattice, we find, depending on parameters, evidence for both continuous phase transitions in the universality class of directed percolation with infinitely many absorbing states and for first-order behavior.

To understand this behavior, we have developed a stochastic model generalizing the Domany-Kinzel cellular automaton. In this model, the active sites can emit solitons and by colliding, the solitons can create new active sites. Simulations of this model, together with the appropriate meanfield theory, support the existence of both continuous and discontinuous transitions. With the stochastic model, one can look at the behavior on much larger length and time scales. One, thereby, discovers that there is a whole range of parameters, where the active states close and above the apparent discontinuous transition are actually metastable, and will 
finally, decay when a sufficiently large droplet nucleates. There is, however, a larger value for the critical parameter where such inactive droplets shrink, and this value could constitute the "true" transition value.

The metastable regime appears very clearly over a surprisingly long range of intermediate time scales, and would thus be relevant in the interpretation of experiments. We further show that this feature can lead to long powerlike transients displaying nonuniversal "critical exponents," and we believe that such transients are the origin of the observed nonuniversality in the transition to spatiotemporal intermittency.

\section{ACKNOWLEDGMENT}

R.M. gratefully acknowledges support form "Stichting Fundamenteel Onderzoek der Materie” (FOM).
[1] E. Domany and W. Kinzel, Phys. Rev. Lett. 53, 311 (1984).

[2] T. Bohr, M.H. Jensen, G. Paladin, and A. Vulpani, Dynamical Systems Approach to Turbulence (Cambridge University Press, Cambridge, 1998).

[3] H. Chaté, Nonlinearity 7, 185 (1994); M. van Hecke, Phys. Rev. Lett. 80, 1896 (1998).

[4] P. Grassberger and T. Schreiber, Physica D 50, 177 (1991).

[5] H. Chaté and P. Manneville, Physica D 32, 409 (1988).

[6] T. Bohr, M. van Hecke, R. Mikkelsen, and M. Ipsen, Phys. Rev. Lett. 86, 5482 (2001).

[7] H. Chaté and P. Manneville, Phys. Rev. Lett. 58, 112 (1987).

[8] H. Chatéin, in Spontaneous Formation of Space-Time Structures and Criticality, edited by T. Riste and D. Sherrington (Kluwer, Dordrecht, 1991), p. 273.

[9] A. Novick-Cohen and G.I. Sivashinsky, Physica D 20, 237 (1986).

[10] S. Ciliberto and P. Bigazzi, Phys. Rev. Lett. 60, 286 (1988); F. Daviaud, M. Dubois, and P. Bergé, Europhys. Lett. 9, 441 (1989); M. Rabaud, S. Michalland, and Y. Couder, Phys. Rev. Lett. 64, 184 (1990); S. Michalland and M. Rabaud, Physica D 61, 197 (1992); P. Rupp, R. Richter, and I. Rehberg, e-print cond-mat/0211137.

[11] Y. Pomeau, Physica D 23, 3 (1986).

[12] G. Grinstein and M.A. Muñoz, in Fourth Granada Lectures in Computational Physics, edited by P. Garrido and J. Marro, Lecture Notes in Physics, Vol. 493 (Springer, Berlin, 1997), p. 223.

[13] H. Hinrichsen, Adv. Phys. 49, 815 (2000).
[14] P. Grassberger, Z. Phys. B: Condens. Matter 47, 365 (1982).

[15] H.K. Janssen, Z. Phys. B: Condens. Matter 42, 151 (1981).

[16] J. Rolf, T. Bohr, and M.H. Jensen, Phys. Rev. E 57, R2503 (1998).

[17] M. Henon, Commun. Math. Phys. 50, 69 (1976).

[18] J.M. Houlrik, I. Webman, and M.H. Jensen, Phys. Rev. A 41, 4210 (1990); J.M. Houlrik and M.H. Jensen, in Theory and Applications of Coupled Maps Lattices, edited by K. Kaneko (Wiley, New York, 1993).

[19] M.A. Muñoz, R. Dickman, A. Vespignani, and S. Zapperi, Phys. Rev. E 59, R6175 (1999).

[20] P. Grassberger and A. de la Torre, Ann. Phys. (N.Y.) 122, 373 (1979).

[21] J.F.F. Mendes, R. Dickman, M. Henckel, and M.C. Marques, J. Phys. A 27, 3019 (1994).

[22] M.A. Munoz, G. Grinstein, and Y. Tu, Phys. Rev. E 56, 5101 (1997); M.A. Munoz, G. Grinstein, and R. Dickman, J. Stat. Phys. 91, 541 (1998).

[23] P. Grassberger, H. Chaté, and G. Rousseau, Phys. Rev. E 55, 2488 (1997).

[24] We have not found any strong indication for the variation of the critical $p_{c}$ with background state [22].

[25] J.R de Bruyn and Lihong Pan, Phys. Rev. E 47, 4575 (1993).

[26] K.R. Elder, J.D. Gunton, and N. Goldenfeld, Phys. Rev. E 56, 1631 (1997); M. Paniconi and K.R. Elder, ibid. 56, 2713 (1997).

[27] N.J. Balmforth, Annu. Rev. Fluid Mech. 27, 335 (1995). 